\title{
Action spéciale pour les 50-65 ans
}

\section{Nouveau placement de capital innovatif et sécurisé}

Lors d'un placement I'on est généralement confronté au choix suivant: sécurité ou rendement? La solution innovative Helvetia Value Trend vous offre toutefois les deux. L'Helvetia vous garantit le remboursement de votre capital et un intérêt minimal après 10 ans. De plus, pendant la durée du placement, vous bénéficiez avec votre capital d'une stratégie indexée laquelle couvre les marchés principaux telles qu'actions, obligations et matières premières. Si à la fin de la période de placement le développement des valeurs s'avérait plus élevé que le montant garanti initialement, vous recevez également ladite valeur supplémentaire.

\section{Paiement sans imposition fiscale}

Tous les clients qui ont entre 50 et 65 ans au 12 décembre 2011 peuvent profiter du paiement non-soumis fiscalement.

Exemple de calcul: femme, 55 ans, investition CHF 100000.-

\begin{tabular}{l|l}
\hline Paiement garanti & CHF 111026.- \\
\hline Paiement avec 3\% p. a. * & CHF 125386.- \\
\hline Paiement avec 4\% p. a. * & CHF 138106.- \\
\hline Paiement avec 5\% p. a. * & CHF 151974.- \\
\hline
\end{tabular}

* Développement de la valeur non-garantie par la stratégie indexée

\section{Le principal en un coup d'œil}

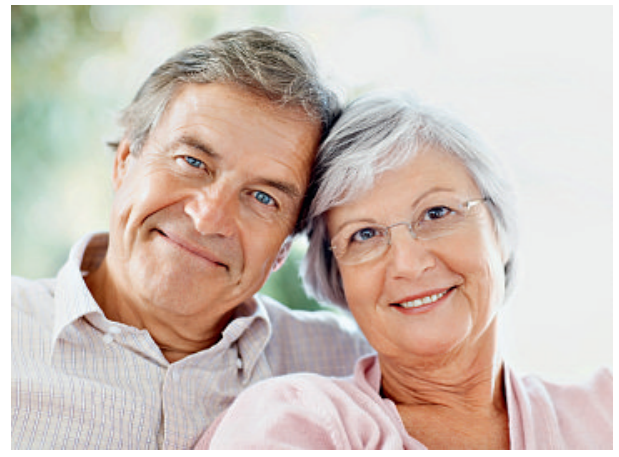

$\rightarrow$ Helvetia Value Trend est limitée dans le temps et valeur et est disponible jusqu'au 9.12.11 au plus tard.

$\rightarrow$ La durée du contrat est de 10 ans.

$\rightarrow$ Le début du contrat est fixé au 12.12.2011. Les paiements anticipés sont honorés d’un intérêt de $2 \%$.

$\rightarrow$ Le droit de timbre de $2,5 \%$ vous est offert!

$\rightarrow$ Vous ne pouvez qu'être gagnant!

Adressez-moi encore aujourd'hui une offre personnelle et plus d'informations sur cette solution innovative.

\section{Talon réponse}

Prénom / Nom

Adresse

NPA / Lieu

Date de naissance

Téléphone privé / cabinet

Atteignable le plus facilement (heure)

Adresse e-mail

Investition

CHF

Prière de $\mathrm{m}^{\prime}$ envoyer une offre Helvetia Value Trend.

Je désire un conseil personnalisé.

Prière de $\mathrm{m}^{\prime}$ appeler.

Je m'intéresse à:

Caisse-maladie

Pilier $3 a$

Planification financière
Caisse de pension LPP

O Responsabilité civile prof.

Protection juridique



Roth Gygax \& Partner AG $\square$ Service de coordination Moosstrasse 2 - 3073 Gümligen

Téléphone 0319595000 a Fax 0319595010 mail@fmhinsurance.ch $\mathbf{w}_{\text {www.fmhinsurance.ch }}$ 